\title{
STOCHASTIC DERIVATION OF THE DIRAC EQUATION IN TERMS OF A FLUID OF SPINNING TOPS ENDOWED WITH RANDOM FLUCTUATIONS AT THE VELOCITY OF LIGHT
}

\author{
N. CUFARO PETRONI \\ Istituto di Fisica doll'Università. Bari, Ital.' \\ and Istituto Nazionale di Fisica Nucleare. Sezione di Bari. Bari. Italy. \\ and \\ J.P. VIGIER \\ Fquipe de Recherche Associéc au CNRS no. 533. Institut H. Poincare. 75231 Patis Cides 0.5. France
}

Received 23 October 1980

\begin{abstract}
If one analyzes the stochastic behaviour of classical "rigid" tops imbedded in Dirac's aether (relativistic thermostat) one obtains (for random jumps at the velocity of light) a probability distribution corresponding to the I eynman-Gell-Mann equation for relativistic spin $1 / 2$ particles.
\end{abstract}

The aim of the present letter is to complete a progran of stochastic derivations of the basic quantummechanical equations with a derivation of the Dirac equation in its Feynman -Gell-Mann form [1]. This is necessary in the stochastic interpretation of quantum mechanics (SIQM) [2-7] for a mathematical and a physical reason.

The mathematical reason is that according to the Cartan-Pauli demonstration [ $8-10]$ there are only three possible basic two-dimensional spin subgroups (which preserve two-dimensional planes). Kinematically they correspond to purely Lorentz $(J=0)$, purely spatial $(J=1)$ and light-cone tangent $(J=1 / 2)$ subrotations in $S O(3,1)$. Mathematically [11] they correspond to the $D(0), D(1)$, and $D(1 / 2)$ representations of the rotation group imbedded in the general representation $\mathrm{D}(1 / 2,1 / 2)$ of the Lorent $z$ group. The usual quantization of their three classical counterparts indeed yields the usual wave equations [10].

The physical reason is that, if one accepts Einstein's idea of a subquantal covariant chaotic vacuum (materialized for example in Dirac's aether model [12.13]), one should be able to deduce (as has already been done in various equivalent ways $[4,5,14])$ not only $J=0$ and $J=1$ equations but also the $J=1 / 2$ equation.
Indeed the proponents of SIQM have attempted, starting in general from a covariant classical (non quantized) electromagnetic vacuum (SED), to derive the Schrödinger and Pauli equations [15]. These important attempts have certainly widened our understanding of the problem, but SED (because it contains no photons) not only contradicts experimental facts [16], but now meets with theoretical troubles in the stability of hydrogen orbits $|17,18|$.

Our starting point is different: we stant with Dirac's aether model recently enlarged to spin [13] by one of us (J.P.V.). a model which implies [5] stochastic motions at the velocity of light. As one now knows this model justifies the validity of Nelson's stochastic equations given in a relativistic form by Guerra and Ruggiero (GR) [19| and Vigier |4]. The application of these markovian processes represented by Nelson's equation in their GR form tos

(a) spin 0 , i.e. extended particles labelled by a position $x_{\mu}(\tau)$ and an invariant density $\rho^{2}(x)$, and

(b) spin 1. i.e. Weyssenhof particles labelled by at position $x_{\mu}(\tau)$ and a complex four-vector $a_{\mu}(x)$ (with $\left.\rho^{2}=a_{\mu}^{*} a^{\mu}\right)$. can now be extended to classical "rigid" tops labelled by a position $x_{\mu}(\tau)$ and a density $\rho^{2}$ of Einstein--Mayer 
orthogonal tetrads of axis $a_{\mu}^{(\xi)}$ represented [20] by a normalized four-component spinor $\psi$. To follow Dirac's aether model we shall of course introduce in our model a density representing a mixture of tops with opposite chiralities (i.e. represented by left and right handed tetrads) which represent a top (particle) and anti-top (antiparticle) mixture [5]. This means that our tops in their stochastic jumps, can move in the forward (particle) or backward (antiparticle) time direction, as introduced in our Markov justification of the Klein-Gordon statistics [5].

We come now to our derivation and we introduce the GR formalism [19]:

$D=\partial / \partial \tau+b_{\nu} \partial^{\nu}, \quad b_{\nu}=D x_{\nu}(\tau)$,

$\delta D=-(2 m)^{-1} \square+\delta b_{\nu} \partial^{\nu}, \quad \delta b_{\nu}=\delta D x_{\nu}(\tau)$,

$\hbar=c=1$, and we suppose that the drift and the stochastic velocities $b_{\mu}$ and $\delta b_{\mu}$ can be derived from

$b^{\mu}=\left(A^{\mu}+\partial^{\mu}(1)\right) / m$,

$\delta b^{\mu}=-(2 m)^{-1} \partial^{\mu} \ln \rho^{2}=-m^{-1}\left(\partial^{\mu} \rho\right) \rho^{-1}$,

where $A_{\mu}(x)$ is the vector potential of an external field, and $\Phi(x, \tau)$ and $\rho(x)$ are real diagonal $4 \times 4$ matrices. We also require that $\rho$ is non singular and that $\rho^{2}$ is a trace invariant matrix which plays the role of a density matrix (but not a definite positive one because of the presence of particles and antiparticles).

The classical covariant stochastic equation and the continuity equation for our "rigid" top in an external field are now

$m(D D-\delta D \delta D) x^{\mu}(\tau)=b_{\nu} F^{\nu \mu}+(4 m)^{-1} \partial^{\mu} \sigma_{\nu \lambda} F^{\nu \lambda}$,

$\partial^{\nu}\left(b_{\nu} \rho^{2}\right)=0$,

where $\sigma^{\mu \nu}=\frac{1}{2} \mathrm{i}\left[\gamma^{\mu}, \gamma^{\nu}\right]$ are the generators of the spinor transformations determined by the Lorentz transformations. We will use in the following the $\gamma$-matrix notation of Bjorken and Drell [21]. Of course eqs. (3) and (4) are $4 \times 4$ matrix equations. From eqs. (1) and (2) we have for eq. (3);

$$
\begin{aligned}
\partial^{\mu} & {\left[(2 m)^{-1}(\square \rho) \rho^{-1}-\partial \Phi / \partial \tau-\left(A_{\nu}+\partial_{\nu} \Phi\right)\left(A^{\nu}+\partial^{\nu} \Phi\right) / 2 m\right.} \\
& \left.+(4 m)^{-1} \sigma_{\nu \lambda} F^{\nu \lambda}\right]=0,
\end{aligned}
$$

which shows that the term in the brackets is constant. If we pose this constant equal to zero, by rearranging the energy scale, eq. (5) takes the following form:

$$
\begin{gathered}
\left(\square-2 m \partial \Phi / \partial \tau-\partial^{\nu} \Phi \partial_{\nu} \Phi-A_{\nu} A^{\nu}\right. \\
\left.-2 A_{\nu} \partial^{\nu} \Phi+\frac{1}{2} \sigma_{\mu \nu} F^{\mu \nu}\right) \rho=0 .
\end{gathered}
$$

Similarly for eq. (4) we have from eqs. (1) and (2):

$\left(2 \partial_{\nu} \Phi \partial^{\nu}+\square \Phi+\partial_{\nu} A^{\nu}+2 A_{\nu} \partial^{\nu}\right) \rho=0$.

If we now make a linear combination of eqs. (6) and (7) with coefficients 1 and i, we have after multiplication by $\exp (\mathrm{i} \Phi)$ :

$$
\begin{aligned}
& {\left[\left(\square+2 \mathrm{i} \partial^{\nu} \Phi \partial_{\nu}+\mathrm{i} \square \Phi-\partial_{\nu} \Phi \partial^{\nu} \Phi-A_{\nu} A^{\nu}\right.\right.} \\
& \left.\quad+\mathrm{i} \partial_{\nu} A^{\nu}+2 \mathrm{i} A^{\nu} \partial_{\nu}-2 A_{\nu} \partial^{\nu} \Phi\right) \rho \\
& \left.\quad+\frac{1}{2} \sigma_{\mu \nu} F^{\mu \nu} \rho-2 m(\partial \Phi / \partial \tau) \rho\right] \exp (\mathrm{i} \Phi)=0,
\end{aligned}
$$

which finally takes the following synthetic form:

$$
\begin{gathered}
{\left[\left(\mathrm{i} \partial_{\nu}-A_{\nu}\right)\left(\mathrm{i} \partial^{\mu}-A^{\nu}\right)-2 \mathrm{i} m \partial / \partial \tau\right.} \\
\left.-\frac{1}{2} \sigma_{\mu \nu} F^{\mu \nu}\right] \exp (\mathrm{i} \Phi) \rho=0 .
\end{gathered}
$$

If we now multiply this matrix equation with an arbitrary constant ( $x$ independent) spinor $w$ and if we pose $\phi(x, \tau)=\exp (\mathrm{i} \Phi) \rho w$ we have

$$
\begin{gathered}
{\left[\left(\mathrm{i} \partial_{\nu}-A_{\nu}\right)\left(\mathrm{i} \partial^{\nu}-A^{\nu}\right)-2 \mathrm{i} m \partial / \partial \tau\right.} \\
\left.-\frac{1}{2} \sigma_{\mu \nu} F^{\mu \nu}\right] \phi(x, \tau)=0
\end{gathered}
$$

which, with the positions

$\Phi(x, \tau)=S(x)-\frac{1}{2} m \tau, \quad \psi(x)=\exp (\mathrm{i} S) \rho w$,

gives exactly the Feynman-Gell-Mann equation

$\left[\left(\mathrm{i} \partial_{\nu}-A_{\nu}\right)\left(\mathrm{i} \partial^{\nu}-A^{\nu}\right)-\frac{1}{2} \sigma^{\mu \nu} F_{\mu \nu}\right] \psi(x)=m^{2} \psi(x)$.

The connection between the $p^{2}$ matrix and the 4 component Dirac spinors $\psi$ is clarified if we consider the four spinors $\psi^{r}=\exp (\mathrm{i} S) \rho w^{r}$ where $w^{r}(r=1,2$, $3,4)$ are constant spinors such that (for $\bar{w}^{r}=w^{r+} \gamma_{0}$ ) $\bar{w}^{r} w^{r^{\prime}}=\epsilon_{r} \delta_{r r^{\prime}}, \quad \epsilon_{r}=+1, r=1,2$, $\sum_{r} \epsilon_{r} w^{r} \bar{w}^{r}=1, \quad=-1, r=3,4$.

For example we could take

$w_{1}=\left(\begin{array}{l}1 \\ 0 \\ 0 \\ 0\end{array}\right), \quad w_{2}=\left(\begin{array}{l}0 \\ 1 \\ 0 \\ 0\end{array}\right), \quad w_{3}=\left(\begin{array}{l}0 \\ 0 \\ 1 \\ 0\end{array}\right), \quad w_{4}=\left(\begin{array}{l}0 \\ 0 \\ 0 \\ 1\end{array}\right)$ 
It is now easy to show that

$\rho^{2}=\sum_{r} \epsilon_{r} \psi^{r} \bar{\psi}^{r}, \quad \operatorname{tr} \rho^{2}=\sum_{r} \epsilon_{r} \bar{\psi}^{r} \psi^{r}$,

are coherent with our requirement of a trace invariant $\rho^{2}$ matrix.

We conclude with two remarks. The first remark is that our (to spin) extended aether model of Dirac [13] imposes different Markov processes on different classical objects which are thus described by different wave equations, i.e. the usual Klein-Gordon, Proca and Dirac equations. In that sense SIQM goes beyond the Copenhagen interpretation of quantum mechanics (CIQM) which introduces them a priori without theoretical justification.

The second remark is that the use of a real material subquantal aether implies predictions not contained in CIQM. If particles indeed behave as oscillators moving in phase with a surrounding wave of oscillations (somewhat like a plane flying at Mach-1 in its surrounding sound wave), the corresponding objective reality of de Broglie's waves opens the way to new types of experiments (proposed by Garuccio and Vigier [22] and Fitchard [23]) in which SIQM and CIQM predict theoretically different testable results. The decision on the Bohr-Einstein controversy thus now rests on the shoulders of experimental physicists.

The authors are grateful to Professurs A.M. Cetto, L. de la Peña-Auerbach, Z. Marić, G. Živanović.

F. Selleri and A. Garuccio for helpful discussions. They are also grateful to the French CNRS and Italian CNR for grants which made this research possible.

\section{References}

[1] R.P. leynman and M. Gell-Mann, Phys. Rev. 109 (1958) 193.

[2] D. Bohm and J.P. Vigier, Phys. Rev. 96 (1954) 208; 109 (1958) 1882.

[3] F. Nelson, Phys. Rev. 150 (1966) 1079.

[4] J.P. Vigier, Lett. Nuovo Cimento 24 (1979) 258, 265.

[5] N. Cufaro Petroni and J.P. Vigier, Int. J. Theor. Phys. $18(1979) 807$.

[6] N. Cufaro Petroni and J.P. Vigier, Phys. Lett. 73 A (1979) 289.

[7] L. de Broglie, La thermodynamique de la particule isolée (Gauthier Villars, Paris, 1964).

[8] L. Cartan. The theory of spinors (Hermann, Paris, 1966).

[9] W. Pauli, Theory of relativity (Pergamon, London, 1958).

[10] Ch. Jenech, M. Moles and J.P. Vigier, Lett. Nuovo Cimento 24 (1979) 56.

[11] P. Hillion and J.P. Vigier, Nucl. Phys. 16 (1960) 360.

[12] P.M.A. Dirac, Nature 168 (1951) 906.

[13] J.P. Vigier, De Broglie's waves in Dirac's aether, Lett. Nuovo Cimento, to be published.

[14] W. Lehr and J. Park, J. Math. Phys. 18 (1977) 1235.

[15] L. de la Peña-Auerbach, J. Math. Phys. 12 (1971) 453.

[16] J.f. Clauser, in: Coherence and quantum optics, eds. L. Mandel and I.. Wolf (Plenum, New York, 1973).

[17] P. Claverie and 1\%. Soto, Non recurrence of stochastic process for the hydrogen atom problem in stochastic electrodynamics, to be published.

[18| P. Claverie, L. Pesquera and I. Soto, Existence of a constant stationary solution for the hydrogen atom problem in stochastic electrodynamics, to be published.

[19] I. Guerra and P. Ruggiero. Lett. Nuovo Cimento 23 (1978) 529 .

[20] D. Gutkowski, M. Moles and J.P. Vigier, Nuovo (imento $39 \mathrm{~B}(1977) 193$.

[21] J.D. Bjorken and S.D. Drell, Relativistic quantum mechanics (McCiraw-Hill, New York, 1964).

[22] A. Garuccio and J.P. Vigier, Possible experimental test of the causal stochastic interpretation of quantum mechanics. Physical reality of de Broglie's waves, Found. Phys. (Oct. 1980).

[23] F.E. Fitchard, Found. Phys. 9 (1979) 525 